\title{
Increased expression of monocarboxylate transporters 1,2 , and 4 in colorectal carcinomas
}

\author{
Céline Pinheiro • Adhemar Longatto-Filho • \\ Cristovam Scapulatempo $\cdot$ Luísa Ferreira • \\ Sandra Martins • Luc Pellerin • Mesquita Rodrigues • \\ Venancio A. F. Alves • Fernando Schmitt • \\ Fátima Baltazar
}

Received: 10 September 2007 /Revised: 5 November 2007 / Accepted: 6 December 2007 / Published online: 10 January 2008

(C) Springer-Verlag 2007

\begin{abstract}
Tumour cells are known to be highly glycolytic, thus producing high amounts of lactic acid. Monocarboxylate transporters (MCTs), by promoting the efflux of the accumulating acids, constitute one of the most important mechanisms in the maintenance of tumour intracellular $\mathrm{pH}$.
\end{abstract}

C. Pinheiro $\cdot$ A. Longatto-Filho $\cdot$ L. Ferreira $\cdot$ S. Martins $\cdot$

F. Baltazar $(\square)$

Life and Health Sciences Research Institute (ICVS),

School of Health Sciences, University of Minho,

Campus de Gualtar,

4710-057 Braga, Portugal

e-mail: fbaltazar@ecsaude.uminho.pt

\author{
A. Longatto-Filho \\ Instituto Adolfo Lutz, \\ São Paulo, Brazil \\ C. Scapulatempo • V. A. F. Alves \\ Department of Pathology, \\ University of São Paulo School of Medicine, \\ São Paulo, Brazil \\ S. Martins $\cdot$ M. Rodrigues \\ S. Marcos Hospital, \\ Braga, Portugal \\ L. Pellerin \\ Faculty of Biology and Medicine, University of Lausanne, \\ Lausanne, Switzerland \\ F. Schmitt \\ Institute of Pathology and Immunology of University of Porto \\ (IPATIMUP), \\ Porto, Portugal \\ F. Schmitt \\ Medical Faculty of the University of Porto, \\ Porto, Portugal
}

Since data concerning MCT expression in colorectal carcinomas (CRC) are scarce and controversial, the present study aimed to assess the expressions of MCT1, 2, and 4 in a well characterized series of CRC and assess their role in CRC carcinogenesis. CRC samples (126 cases) were analyzed for MCT1, MCT2, and MCT4 immunoexpression and findings correlated with clinico-pathological parameters. Expression of all MCT isoforms in tumour cells was significantly increased when compared to adjacent normal epithelium. Remarkably, there was a significant gain of membrane expression for MCT1 and MCT4 and loss of plasma membrane expression for MCT2 in tumour cells. Plasma membrane expression of MCT1 was directly related to the presence of vascular invasion. This is the larger study on MCT expression in CRC and evaluates for the first time its clinico-pathological significance. The increased expression of these transporters suggests an important role in CRC, which might justify their use, especially MCT1 and MCT4, as targets in CRC drug therapy.

Keywords Monocarboxylate transporter.

Colorectal carcinoma

\section{Introduction}

Highly proliferative cancer cells maintain a high rate of glycolysis, producing large amounts of acids, mainly lactic acid. Despite this fact, only the interstitial $\mathrm{pH}$ of tumours is low, while the intracellular $\mathrm{pH}$ of tumours is either normal or higher than that of normal tissues $[3,10]$. In order to prevent apoptosis by cellular acidosis, tumour cells increase proton efflux through $\mathrm{pH}$ regulators, such as proton pumps, sodium-proton exchangers, bicarbonate transporters, and 
monocarboxylate transporters (MCTs), which are described to be up-regulated in tumour cells [11].

MCTs play a central role in cellular metabolism and are essential for transport of monocarboxylates, such as lactate, across the plasma membrane. Based on sequence homologies, 14 MCT family members were identified, although only a few have been functionally characterized. MCT1MCT4 isoforms are proton symporters, which have lactate as a common substrate, each isoform exhibiting different biochemical properties [9]. Substrate specificity has been associated with the tissue distribution of each transporter, depending on their metabolic activity: MCT1 has an ubiquitous distribution [6], while MCT2, having a high affinity for the substrate, is found in tissues that use lactate as a substrate (e.g., liver) [2]. MCT4, which is a lowaffinity transporter, is present in highly glycolytic tissues (e.g., slow-twitch muscle) [4] and MCT3 is exclusively found in the retinal pigment epithelium and in the choroid plexus epithelium [16].

Using in vitro and ex vivo models, Wahl et al. [19] demonstrated that MCTs are major players in the regulation of $\mathrm{pH}$ in melanoma cells and proposed that MCT inhibitors may be particularly effective against malignant melanoma. There are also evidence for the upregulation of MCTs in other tumours such as alveolar sarcoma of soft tissues [13], high grade glial neoplasms [7, 15], and colorectal carcinomas (CRC) [12].

Lactate release from tumour cells is a common endpoint of several metabolic alterations, raising the hypothesis of an association with tumour progression [21]. Some evidence support this hypothesis: low interstitial $\mathrm{pH}$ is associated with the upregulation of various angiogenic molecules, which support tumour growth, invasion, and metastasis such as vascular endothelial growth factor [8]. Lactate accumulation has also been associated with metastization and lower disease-free and overall survival in squamous cell carcinomas of the uterine cervix and head and neck carcinomas $[1,20]$. Targeting MCT activity would not only induce apoptosis due to cellular acidosis, but would also lead to reduction in tumour angiogenesis, invasion, and metastasis. Thus, MCTs can constitute an attractive target for cancer therapy.

Data concerning expression of MCTs in CRC are scarce and controversial. While Lambert et al. [14] described a decrease in MCT1 expression during transition to malignancy, Koukourakis et al. [12] reported an increase in MCT1 expression in tumour cells, which is supported by the metabolic alterations induced by anaerobic glycolysis. The latter study also assessed the expression of MCT2 and MCT4, finding a strong cytoplasmic expression of MCT2 in cancer cells, but a weak expression of MCT4 in the tumour environment, suggesting a minimal role of this $\mathrm{MCT}$ isoform in $\mathrm{CRC}$ metabolic homeostasis.
Our study aimed to contribute to the elucidation of the role of MCTs in CRC, by assessing the immunohistochemical expression of the MCT isoforms 1, 2, and 4 in a large and well characterized series of CRC and evaluating its clinico-pathological significance.

\section{Materials and methods}

A series of formalin-fixed, paraffin-embedded tissues from $126 \mathrm{CRC}$ was retrieved from the files of the Medical School of the University of São Paulo (Brazil). Tissue samples were analyzed for MCT1, MCT2, and MCT4 immunohistochemical expression using specific antibodies. Comparison of MCT expression in tumour vs normal cells was also possible since, for most cases, the same paraffin section contained both tumour and normal colonic epithelium. MCT immunohistochemical expression was correlated with the available clinico-pathological data, which included: age, gender, tumour size, macroscopic presentation, histological type, tumour localization, depth of invasion, vascular invasion, nodal metastasis, and TNM classification. Normal colorectal mucosa, liver, and skeletal muscle samples were obtained prospectively from patients undergoing surgery: colorectal mucosa and abdominal wall skeletal muscle were obtained from a patient with diagnosis of diverticulosis; liver was from a patient with a diagnosis of metastatic colon carcinoma. Informed written consent was obtained from the individuals prior to removal of the tissue.

\section{Western blotting}

The specificity of the antibodies used in immunohistochemistry was evaluated by Western blotting. Tissue samples from human colon, liver, and muscle were used as positive controls for the expression of MCT1, 2, and 4, respectively. Briefly, fresh samples were collected, homogenized in a lysis buffer and then centrifuged at 6,000 $\mathrm{rpm}, 20 \mathrm{~min}$ at $4^{\circ} \mathrm{C}$. The supernatants were collected and protein concentrations were determined by the method of Lowry. Protein was separated on a $10 \%(w / v)$ polyacrylamide gel and transferred onto a nitrocellulose membrane (Amersham Pharmacia Biotech, Piscataway, NJ, USA). Membranes were blocked with $1 \%$ Tween, $10 \%$ milk, and $1 \%$ bovine serum albumin in TBS for $1 \mathrm{~h}$. Membranes were then incubated overnight at $4{ }^{\circ} \mathrm{C}$ with primary polyclonal antibodies for MCT1 (AB3538P, Chemicon International, Temecula, CA, USA), MCT2 (sc-14926, Santa Cruz Biotechnology, Santa Cruz, CA, USA), and MCT4 (AB3316P, Chemicon International), diluted 1:200 for MCT1 and MCT4, and 1:100 for MCT2. Membranes were then incubated with the secondary 
antibody coupled to horseradish peroxidase (SantaCruz Biotechnology) and bound antibodies were visualized by chemiluminescence (Supersignal West Femto kit, Pierce, Rockford, IL, USA).

\section{Immunohistochemistry}

Immunohistochemistry was performed according to avidinbiotin-peroxidase complex principle (R.T.U. VECTASTAIN Elite ABC Kit (Universal), Vector Laboratories, Burlingame, CA, USA), with the primary antibodies for MCT1, MCT2, and MCT4, used in Western blotting, diluted 1:200. Briefly, deparaffinized and rehydrated sections were incubated in $0.3 \%$ hydrogen peroxide for $30 \mathrm{~min}$, to inactivate endogenous peroxidases, and washed in PBS. Antigen retrieval was performed by immersing slide-mounted sections in $0.01 \mathrm{M}$ citrate buffered solution (pH 6.0) and microwaving (600 W) for $15 \mathrm{~min}$ then washed in PBS. Tissue sections were then incubated with a protein blocking solution for $20 \mathrm{~min}$ and incubated with the primary antibody overnight at room temperature. Sections were then sequentially washed in PBS and incubated with biotinylated secondary antibody for $30 \mathrm{~min}$, R.T.U. Vectastain ${ }^{\circledR}$ Elite $\mathrm{ABC}$ reagent for $45 \mathrm{~min}$ at $37^{\circ} \mathrm{C}$ and developed with 3,3'-diamino-benzidine (DAB+ Substrate System, DakoCytomation, Carpinteria, CA, USA) for 10 min. Negative controls were performed by using the adequate serum controls for the primary antibodies used (N1699 and X0907, DakoCytomation, Carpinteria, CA, USA). Normal colon, kidney, and skeletal muscle tissues were used as positive controls for MCT1, MCT2, and MCT4, respectively. Tissue sections were counterstained with haematoxylin and permanently mounted.

\section{Immunohistochemical evaluation}

Sections were scored semi-quantitatively for immunoreaction extension as follows: 0: $0 \%$ of immunoreactive cells; 1 : $<5 \%$ of immunoreactive cells; $2: 5-50 \%$ of immunoreactive cells; and 3: $>50 \%$ of immunoreactive cells. Also, intensity of staining was scored semi-qualitatively as 0 : negative; 1 : weak; 2: intermediate; and 3: strong. Immunoreaction final score was defined as the sum of both parameters (extension and intensity), and grouped as negative (0), weak (2), moderate (3), and strong (4-6). For statistical purposes, only moderate and strong immunoreaction final scores were considered as positive. Finally, positive plasma membrane staining was also assessed. Evaluation of MCT immunohistochemical expression was performed blindly by two independent observers and discordant cases were discussed in a double-head microscope in order to determine a final score.

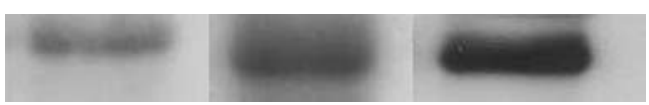

Fig. 1 Western blotting of human tissue lysates for $M C T 1,2$, and 4. Normal colon, liver, and skeletal muscle were used for $M C T 1,2$, and 4 detection, respectively

Statistical analysis

Data were stored and analyzed using the SPSS statistical software (version 14.0, SPSS Inc., Chicago, IL, USA). The comparison of MCT expression between tumour and normal cells as well as the relationship between MCT expressions and the clinico-pathological parameters were examined for statistical significance using Pearson's chisquare $\left(\chi^{2}\right)$ test, with the threshold for significant $p$ values being $<0.05$.

\section{Results}

One hundred and twenty six cases of $\mathrm{CRC}$, corresponding to 56 women and 70 men with a median age of 64 (range 24 95), were analyzed for MCT1, MCT2, and MCT4 immunohistochemical expression. Western blot analyses of normal human tissues, using the antibodies for the three MCT isoforms, revealed protein bands around $50 \mathrm{kDa}$ (Fig. 1), consistent with the molecular weight described for these membrane proteins [7].

Table 1 summarizes the frequency of MCT isoforms 1, 2 , and 4 expressions in tumour cells and normal adjacent epithelium. In positive cases, cytoplasmic staining was always present while the frequency of plasma membrane staining differed for each isoform, being more frequent for

Table 1 Pattern of MCT staining in tumour vs normal adjacent epithelium

\begin{tabular}{lllllll}
\hline \multirow{2}{*}{ Isoform } & $n$ & \multicolumn{2}{c}{ Immunoreaction } & & \multicolumn{2}{c}{ Plasma membrane } \\
& & Positive (\%) & $p$ & & Positive (\%) & $p$ \\
\hline MCT1 & & & $<0.001$ & & $<0.001$ \\
Normal & 86 & 34.9 & & 9.3 & \\
Tumour & 126 & 85.7 & & 51.6 & 0.031 \\
MCT2 & & & 0.017 & & \\
Normal & 84 & 83.3 & & 14.3 & \\
Tumour & 126 & 93.7 & & 5.6 & $<0.001$ \\
MCT4 & & & $<0.001$ & & \\
Normal & 89 & 55.1 & & 9.0 & \\
Tumour & 126 & 96.0 & & 38.1 & \\
\hline
\end{tabular}




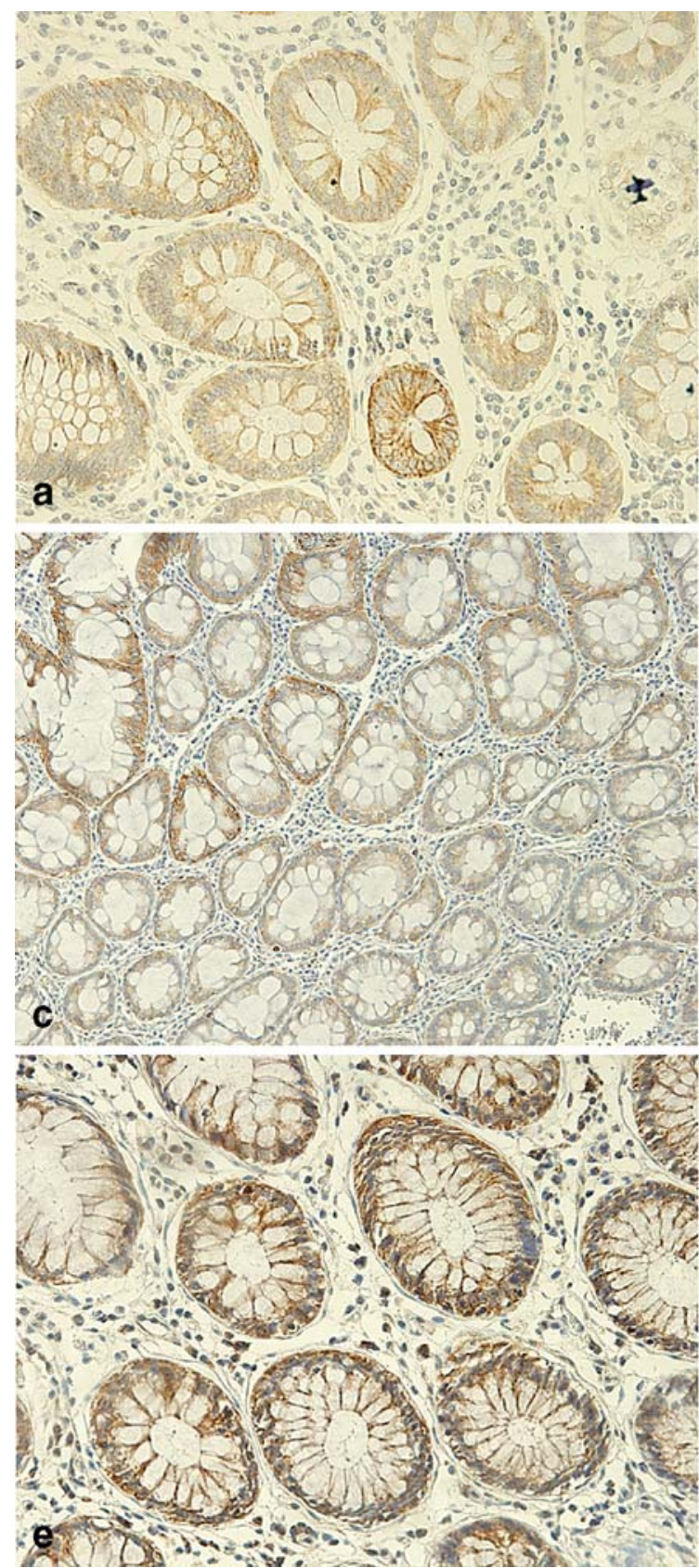

Fig. 2 Immunohistochemical expression of MCT1, 2, and 4 in colon carcinoma samples. Staining in tumour cells (b, d, and f: MCT1, 2, and 4 , respectively) was stronger than in the adjacent normal

MCT1 than for the other isoforms $(51.6 \%$ vs $38.1 \%$ for MCT4 vs 5.6\% for MCT2).

MCT1 immunoexpression in tumour cells was significantly increased, when compared to adjacent normal epithelium $(85.7 \%$ vs $34.9 \%, p<0.001$, Table 1$)$. Importantly, plasma membrane staining was also significantly more frequent in tumour than in normal cells $(51.6 \%$ vs $9.3 \%, p<0.001$, Table 1). Plasma membrane expression of MCT1 (Fig. 2) was directly related to the presence of vascular invasion $(p=0.003$, Table 2$)$.
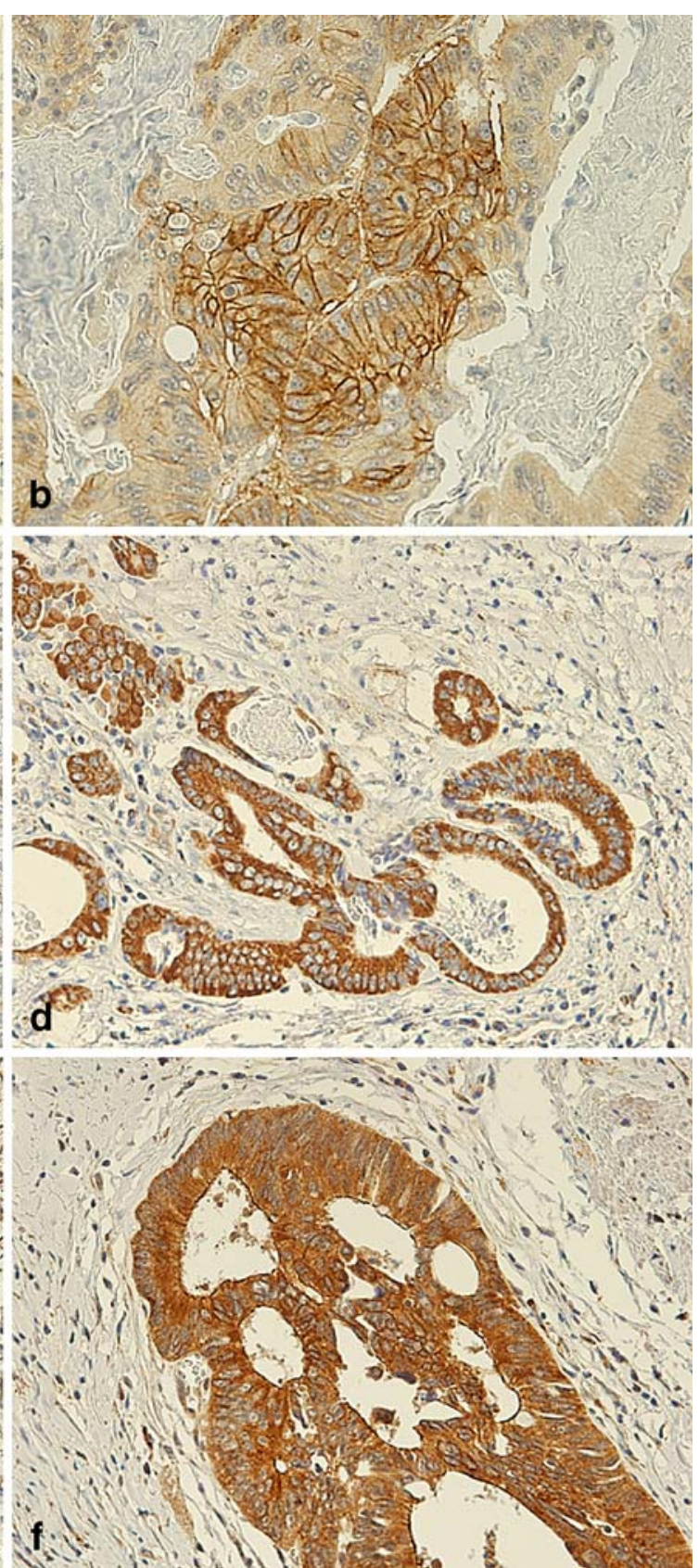

epithelium (a, c, and e: MCT1, 2, and 4, respectively). MCT1 plasma membrane staining (b) was frequently observed in tumour cells

Staining for MCT2 in tumour cells was also more frequent than in the adjacent normal epithelium $(93.7 \%$ vs $83.3 \% ; p=0.017$, Table 1). Remarkably, there was a significant loss of plasma membrane staining in tumour cells $(14.3 \%$ vs $5.6 \% ; p<0.001$, Table 1$)$. Although it was possible to observe "granule" like cytoplasmic staining for all MCT isoforms, this pattern was much more common for MCT2 in tumour cells (Fig. 2).

MCT4 was the most frequently expressed isoform in tumour cells, with $96 \%$ of the cases showing positive 
Table 2 Assessment of correlation between MCTs membrane expression and the clinico-pathological data

\begin{tabular}{|c|c|c|c|c|c|c|c|}
\hline \multirow{3}{*}{ Clinical data } & \multicolumn{7}{|c|}{ Plasma membrane } \\
\hline & \multicolumn{3}{|c|}{ MCT1 } & \multicolumn{2}{|l|}{ MCT2 } & \multicolumn{2}{|l|}{ MCT4 } \\
\hline & $n$ & Positive (\%) & $p$ & Positive (\%) & $p$ & Positive (\%) & $p$ \\
\hline Tumour size $(\mathrm{cm})$ & & & 0.897 & & 0.117 & & 0.572 \\
\hline$<5$ & 53 & 50.9 & & 1.9 & & 35.8 & \\
\hline$\geq 5$ & 71 & 52.1 & & 8.5 & & 40.8 & \\
\hline Macroscopic type & & & 0.826 & & 0.360 & & 0.548 \\
\hline Exophytic & 26 & 46.2 & & 0.0 & & 30.8 & \\
\hline Ulcerative & 49 & 55.1 & & 8.2 & & 40.8 & \\
\hline Infiltrative & 12 & 58.3 & & 0.0 & & 25.0 & \\
\hline Sessile-ulcerated & 39 & 48.7 & & 7.7 & & 43.6 & \\
\hline Histological type & & & 0.563 & & 0.551 & & 0.586 \\
\hline Adenocarcinoma & 111 & 50.5 & & 5.4 & & 38.7 & \\
\hline Mucinous & 10 & 60.0 & & 10.0 & & 30.0 & \\
\hline Localization $^{\mathrm{a}}$ & & & 0.261 & & 0.474 & & 0.923 \\
\hline Right colon & 21 & 42.9 & & 4.8 & & 38.1 & \\
\hline Left colon & 40 & 45.0 & & 2.5 & & 40.0 & \\
\hline Rectum & 61 & 59.0 & & 8.2 & & 36.1 & \\
\hline Invasion & & & 0.251 & & 0.208 & & 0.829 \\
\hline $\mathrm{T} 1+\mathrm{T} 2$ & 22 & 40.9 & & 0.0 & & 36.4 & \\
\hline $\mathrm{T} 3+\mathrm{T} 4$ & 103 & 54.4 & & 6.8 & & 38.8 & \\
\hline Vascular invasion $^{\mathrm{b}}$ & & & 0.003 & & 0.090 & & 0.400 \\
\hline Absent & 57 & 36.8 & & 1.8 & & 42.1 & \\
\hline Present & 69 & 63.8 & & 8.7 & & 34.8 & \\
\hline Nodal metastasis & & & 0.212 & & 0.697 & & 1.000 \\
\hline Absent & 63 & 46.0 & & 4.8 & & 38.1 & \\
\hline Present & 63 & 57.1 & & 6.3 & & 38.1 & \\
\hline TNM & & & 0.408 & & 0.722 & & 0.276 \\
\hline Stage I & 16 & 43.8 & & 0.0 & & 31.3 & \\
\hline Stage II & 35 & 42.9 & & 5.7 & & 40.0 & \\
\hline Stage III & 38 & 60.5 & & 7.9 & & 28.9 & \\
\hline Stage IV & 36 & 55.6 & & 5.6 & & 50.0 & \\
\hline
\end{tabular}

${ }^{a}$ Tumour localization was grouped as follows: right colon — cecum and ascendant colon; left colon—splenic flexure, descendant colon, sigmoid and rectosigmoid.

${ }^{\mathrm{b}}$ Vascular invasion includes both lymph and blood vessel invasion.

staining, and expression was significantly more frequent than adjacent normal epithelium $(p<0.001$, Table 1). Figure 2 shows representative cases of MCT4 positive staining in tumour cells and in adjacent normal epithelium. As observed for MCT1, MCT4 plasma membrane staining in tumour cells was significantly more frequent than in the adjacent normal epithelium ( $p<0.001$, Table 1). Assessment of correlation between MCT4 expression and the clinicopathological data revealed a positive association between MCT4 positivity and small size tumours ( $p=0.049$, Table 3$)$.

\section{Discussion}

Experimental evidence point at MCTs as potential targets for cancer therapy $[5,15,19]$. However, the role of these membrane proteins in solid tumour development and survival is not fully understood. In the present study, we showed an evident increase in MCT immunoexpression in CRC cells when compared to adjacent normal epithelium. Most importantly, we evaluated the correlation between MCT expression in CRC and clinico-pathological data for the first time.

Although MCT1 is described as having a ubiquitous distribution, it appears that this MCT isoform plays an important role in the bidirectional transport of lactate and other short-chain fatty acids across the colonic luminal membrane [17]. Unexpectedly, when analysing MCT expression in the available adjacent normal epithelium, we found that MCT1 was the least expressed isoform. However, since these normal tissue areas are in the surroundings of tumour cells, we cannot rule out the possibility that these areas are under the influence of 
Table 3 Assessment of correlation between MCTs immunoreaction and the clinico-pathological data

\begin{tabular}{|c|c|c|c|c|c|c|c|}
\hline \multirow{3}{*}{ Clinical data } & \multicolumn{7}{|c|}{ Immunoreaction } \\
\hline & \multirow[t]{2}{*}{$N$} & \multicolumn{2}{|l|}{ MCT1 } & \multicolumn{2}{|l|}{ MCT2 } & \multicolumn{2}{|l|}{ MCT4 } \\
\hline & & Positive (\%) & $p$ & Positive (\%) & $P$ & Positive (\%) & $p$ \\
\hline Tumour size $(\mathrm{cm})$ & & & 0.383 & & 0.117 & & 0.049 \\
\hline$<5$ & 53 & 88.7 & & 98.1 & & 100.0 & \\
\hline$\geq 5$ & 71 & 83.1 & & 91.5 & & 93.0 & \\
\hline Macroscopic type & & & 0.267 & & 0.967 & & 0.480 \\
\hline Exophytic & 26 & 76.9 & & 92.3 & & 100.0 & \\
\hline Ulcerative & 49 & 89.8 & & 93.9 & & 93.9 & \\
\hline Infiltrative & 12 & 75.0 & & 91.7 & & 91.7 & \\
\hline Sessile-ulcerated & 39 & 89.7 & & 94.9 & & 97.4 & \\
\hline Histological type & & & 0.509 & & 0.413 & & 0.542 \\
\hline Adenocarcinoma & 111 & 87.4 & & 93.7 & & 96.4 & \\
\hline Mucinous & 10 & 80.0 & & 100.0 & & 100.0 & \\
\hline Localization $^{\mathrm{a}}$ & & & 0.586 & & 0.714 & & 0.900 \\
\hline Right colon & 21 & 81.0 & & 90.5 & & 95.2 & \\
\hline Left colon & 40 & 82.5 & & 95.0 & & 95.0 & \\
\hline Rectum & 61 & 88.5 & & 95.1 & & 96.7 & \\
\hline Invasion & & & 0.220 & & 0.570 & & 0.292 \\
\hline $\mathrm{T} 1+\mathrm{T} 2$ & 22 & 77.3 & & 90.9 & & 100.0 & \\
\hline $\mathrm{T} 3+\mathrm{T} 4$ & 103 & 87.4 & & 94.2 & & 95.1 & \\
\hline Vascular invasion $^{\mathrm{b}}$ & & & 0.942 & & 0.081 & & 0.810 \\
\hline Absent & 57 & 86.0 & & 89.5 & & 96.5 & \\
\hline Present & 69 & 85.5 & & 97.1 & & 95.7 & \\
\hline Nodal metastasis & & & 0.611 & & 1.000 & & 0.648 \\
\hline Absent & 63 & 84.1 & & 93.7 & & 95.2 & \\
\hline Present & 63 & 87.3 & & 93.7 & & 96.8 & \\
\hline TNM & & & 0.610 & & 0.591 & & 0.238 \\
\hline Stage I & 16 & 75.0 & & 87.5 & & 100.0 & \\
\hline Stage II & 35 & 85.7 & & 97.1 & & 91.4 & \\
\hline Stage III & 38 & 86.8 & & 92.1 & & 94.7 & \\
\hline Stage IV & 36 & 88.9 & & 94.4 & & 100.0 & \\
\hline
\end{tabular}

${ }^{a}$ Tumour localization was grouped as follows: right colon—cecum and ascendant colon; left colon—splenic flexure, descendant colon, sigmoid and rectosigmoid.

${ }^{\mathrm{b}}$ Vascular invasion includes both lymph and blood vessel invasion.

tumour microenvironment and could show altered MCT expression. It would then be important to further analyze MCT expression in a series of normal colorectal samples. Data concerning MCT1 expression in solid tumours is scarce and there are conflicting data related to CRC $[12,14$, 17]. Our immunohistochemical findings are in agreement with the results of Koukourakis et al. [12]. Although using different antibodies from this previous study, we were also able to detect a high percentage of positive cases for MCT1 expression as well as MCT1 plasma membrane expression in a high number of cases. However, these findings diverge from previous reports, where the authors described a decrease in MCT1 expression in CRC during transition from normality to malignancy [14, 17]. Concerning the clinico-pathological data, we found a significant correlation between MCT1 plasma membrane staining and vascular invasion. MCT1 plasma membrane localization is essential for transporter activity and, assuming a role in the efflux of accumulating acids, MCT1 activity would lead to extracellular acidification. Then, the positive correlation between MCT1 plasma membrane staining and vascular invasion would be in harmony with previous reports which stated that extracellular acidification induces invasion [10].

Data concerning MCT2 expression in solid tumour is also limited. Contrasting with the results presented by Lambert et al. [14], we found expression of MCT2 in both adjacent normal epithelium and tumour cells in CRC. The staining pattern was cytoplasmic and, in a low number of cases, associated with the plasma membrane. Notably, we found a significant decrease in plasma membrane staining in tumour cells, when comparing to adjacent normal epithelium. This finding is in agreement with the results of Koukourakis et al. [12], who described lack of plasma membrane expression in tumour cells but a strong cyto- 
plasmic expression. The authors hypothesized that this cytoplasmic expression was probably associated with the presence of MCT2 in mitochondrial membranes. Taking into consideration the physiological role of MCT2 in the transport of pyruvate into the mitochondria, one could hypothesize that the highly glycolytic tumour cell would up-regulate MCT2 in an attempt to oxidize the excess pyruvate produced. This process would also contribute to the "removal" of the acid accumulated in the cytoplasm.

Concerning MCT4, we found it to be the most frequently expressed isoform in tumour cells, an expression which was significantly increased when compared to the adjacent normal epithelium. These findings contrast with the results of Lambert et al. [14], who did not find MCT4 expression in colon carcinoma and with the results of Koukourakis et al. [12], who described weak MCT4 expression in the tumour environment. This discrepancy could be related to the specificity of the antibodies used. In our study, the frequency of MCT4 staining in tumour cells was significantly different from the adjacent normal epithelium, which, together with the Western blot results, led us to believe in the specificity of the antibody used. We also found an increase in MCT4 plasma membrane staining in tumour cells when comparing with the adjacent normal epithelium. This fact supports the hypothesis that MCTs are important in the efflux of accumulated acids in tumour cells, specially MCT4 which, due to its low affinity for lactate, would be important in highly glycolytic cells [4]. We can thus postulate that MCT4 presence in the plasma membrane will allow continuous glycolytic rate (by "removing" the end product), prevention of apoptosis due to intracellular acidification and contribution to cancer cell survival. Evaluation of the correlation between MCT4 expression and clinico-pathological data revealed positive associations with tumour size, however the correlation is weak ( $p=0.049)$. Nevertheless, assuming that the glycolysis rate in smaller tumours is higher than in bigger tumours [18], and considering the role of MCT4 in lactate efflux, it is understandable that MCT4 activity would not be so crucial in bigger-sized tumours.

One of the most notable findings in this study was the significant gain of MCT1 and MCT4 plasma membrane expression and loss of MCT2 plasma membrane expression in cancer cells. Since MCTs need to be in the plasma membrane in order to export the accumulating acids in highly glycolytic cells, and taking into consideration the substrate affinities of the different isoforms, MCT1 and MCT4 expression in the membrane is in agreement with the metabolic alterations observed in cancer cells. On the other hand, MCT2, which is involved in the uptake of monocarboxylates into the cells in normal cell metabolism [2], does not appear to have an important role in highly glycolytic cancer cells.
This is the most comprehensive study on MCT expression in CRC and the above findings point to MCTs, specially MCT1 and MCT4, as playing an important role in CRC. MCT aberrant expression might justify future studies using these proteins as targets in CRC drug therapy. So far, however, the level of human toxicity and efficacy of the known MCT inhibitors are not currently known [7] and the search for effective and safe inhibitors would be of great value.

Acknowledgments $\mathrm{CP}$ received a $\mathrm{PhD}$ fellowship from Fundação para a Ciência e Tecnologia (SFRH/BD/27465/2006).

Conflict of interest statement We declare that we have no conflict of interest.

\section{References}

1. Brizel DM, Schroeder T, Scher RL, Walenta S, Clough RW, Dewhirst MW, Mueller-Klieser W (2001) Elevated tumour lactate concentrations predict for an increased risk of metastases in headand-neck cancer. Int J Radiat Oncol Biol Phys 51:349-353

2. Broer S, Broer A, Schneider HP, Stegen C, Halestrap AP, Deitmer JW (1999) Characterization of the high-affinity monocarboxylate transporter MCT2 in Xenopus laevis oocytes. Biochem J 341:529-535

3. Dang CV, Semenza GL (1999) Oncogenic alterations of metabolism. Trends Biochem Sci 24:68-72

4. Dimmer KS, Friedrich B, Lang F, Deitmer JW, Broer S (2000) The low-affinity monocarboxylate transporter MCT4 is adapted to the export of lactate in highly glycolytic cells. Biochem J 1:219-227

5. Fang J, Quinones QJ, Holman TL, Morowitz MJ, Wang Q, Zhao H, Sivo F, Maris JM, Wahl ML (2006) The H+-linked monocarboxylate transporter (MCT1/SLC16A1): a potential therapeutic target for high-risk neuroblastoma. Mol Pharmacol 70:2108-2115

6. Fishbein WN, Merezhinskaya N, Foellmer JW (2002) Relative distribution of three major lactate transporters in frozen human tissues and their localization in unfixed skeletal muscle. Muscle Nerve 26:101-112

7. Froberg MK, Gerhart DZ, Enerson BE, Manivel C, Guzman-Paz M, Seacotte N, Drewes LR (2001) Expression of monocarboxylate transporter MCT1 in normal and neoplastic human CNS tissues. Neuroreport 12:761-765

8. Fukumura D, Xu L, Chen Y, Gohongi T, Seed B, Jain RK (2001) Hypoxia and acidosis independently up-regulate vascular endothelial growth factor transcription in brain tumors in vivo. Cancer Res 61:6020-6024

9. Halestrap AP, Meredith D (2004) The SLC16 gene family-from monocarboxylate transporters (MCTs) to aromatic amino acid transporters and beyond. Pflugers Arch 447:619-628

10. Helmlinger G, Sckell A, Dellian M, Forbes NS, Jain RK (2002) Acid production in glycolysis-impaired tumors provides new insights into tumor metabolism. Clin Cancer Res 8:1284-1291

11. Izumi $\mathrm{H}$, Torigoe $\mathrm{T}$, Ishiguchi $\mathrm{H}$, Uramoto H, Yoshida $\mathrm{Y}$, Tanabe M, Ise T, Murakami T, Yoshida T, Nomoto M, Kohno K (2003) Cellular $\mathrm{pH}$ regulators: potentially promising molecular targets for cancer chemotherapy. Cancer Treat Rev 29:541-549

12. Koukourakis MI, Giatromanolaki A, Harris AL, Sivridis E (2006) Comparison of metabolic pathways between cancer cells and stromal cells in colorectal carcinomas: a metabolic survival role for tumor-associated stroma. Cancer Res 66:632-637

13. Ladanyi M, Antonescu CR, Drobnjak M, Baren A, Lui MY, Golde DW, Cordon-Cardo C (2002) The precrystalline cytoplasmic 
granules of alveolar soft part sarcoma contain monocarboxylate transporter 1 and CD147. AJP 160:1215-1221

14. Lambert DW, Wood IS, Ellis A, Shirazi-Beechey SP (2002) Molecular changes in the expression of human colonic nutrient transporters during the transition from normality to malignancy. Br J Cancer 86:1262-1269

15. Mathupala SP, Parajuli P, Sloan AE (2004) Silencing of monocarboxylate transporters via small interfering ribonucleic acid inhibits glycolysis and induces cell death in malignant glioma: an in vitro study. Neurosurgery 55:1410-1419

16. Philp NJ, Yoon H, Lombardi L (2001) Mouse MCT3 gene is expressed preferentially in retinal pigment and choroid plexus epithelia. Am J Physiol Cell Physiol 280:1319-1326

17. Ritzhaupt A, Wood IS, Ellis A, Hosie KB, Shirazi-Beechey SP (1998) Identification and characterization of a monocarboxylate transporter (MCT1) in pig and human colon: its potential to transport L-lactate as well as butyrate. J Physiol 513:719-732

18. Vaupel P (2004) The role of hypoxia-induced factors in tumor progression. Oncologist 9:10-17

19. Wahl ML, Owen JA, Burd R, Herlands RA, Nogami SS, Rodeck U, Berd D, Leeper DB, Owen CS (2002) Regulation of intracellular $\mathrm{pH}$ in human melanoma: potential therapeutic implications. Mol Cancer Ther 1:617-628

20. Walenta S, Wetterling M, Lehrke M, Schwickert G, Sundfor K, Rofstad EK, Mueller-Klieser W (2000) High lactate levels predict likelihood of metastases, tumor recurrence, and restricted patient survival in human cervical cancers. Cancer Res 60:916-921

21. Walenta S, Schroeder T, Mueller-Klieser W (2004) Lactate in solid malignant tumors: potential basis of a metabolic classification in clinical oncology. Curr Med Chem 11:2195-2204 